\title{
TIMING ERROR DETECTOR USING PARTICLE FILTERING
}

\author{
Tanya Bertozzi ${ }^{* \dagger}$ and Didier Le Ruyet ${ }^{\dagger}$ \\ *DIGINEXT, 45 impasse de la Draille, 13857 Aix en Provence Cedex 3, France, Email: bertozzi@ diginext.fr \\ $\dagger$ CNAM, 292 rue Saint Martin, 75141 Paris Cedex 3, France, Email: leruyet@diginext.fr
}

\begin{abstract}
In this paper we propose a new timing error detector for timing tracking loops inside the Rake receiver in DS-CDMA. Based on a particle filter, this timing error detector jointly tracks the delays of each path of the frequency selective channels. The proposed scheme avoids the drawback of the classical early late gate detector which is not able to separate closely spaced paths. Simulation results show that the proposed detector outperforms the conventional early late gate detector in indoor scenarios.
\end{abstract}

\section{INTRODUCTION}

For DS-CDMA systems, the adapted filter generally used is the Rake receiver. This receiver is composed of a set of correlators associated with the most significant paths of the multipath channels. The outputs of these branches are combined to estimate the transmitted symbols. The performance of the Rake receiver strongly depends on the Timing Error Detector (TED). Indeed, we need to estimate the delay of each path: after the acquisition phase where the number of the most significant paths is evaluated and a coarse determination of the delays is performed, the tracking phase is started. The tracking phase is usually achieved by using an Early Late Gate-TED (ELG-TED) associated with each path. It is well known that the ELG-TED cannot separate the individual paths when they are closer than one chip period from other paths. This scenario corresponds for example to the indoor scenario. Recent researches have been conducted on time tracking in frequency selective fading environments [4] [5].

In this paper we propose to use the Particle Filtering (PF) or Sequential Monte Carlo (SMC) methods for the estimation of the delays of the paths in multipath fading channels. The PF methods [6] represent the most powerful approach for the sequential estimation of the hidden state of a nonlinear dynamic model. The solution to this problem depends on the knowledge of the Posterior Probability Density (PPD) of the hidden state given the observations. Except in a few special cases, it is impossible to calculate analytically a sequential expression of this PPD. It is necessary to adopt numerical approximations. The PF methods allow to approximate iteratively the PPD of the hidden state by weighted points or particles which evolve in the state space. Therefore, these methods provide a discrete approximation of the continuous space of the hidden state.

This paper is organized as follows. In Section 2, we will introduce the system model. Then in Section 3, we will describe the PF-based TED (PF-TED). In Section 4, we will give simulation results. Finally, we will draw a conclusion in Section 5.

\section{SYSTEM MODEL}

We consider a DS-CDMA system sending a complex data sequence $\left\{s_{n}\right\}$. The data symbols are spread by a spreading sequence $\left\{d_{m}\right\}_{m=0}^{N_{s}-1}$ where $N_{s}$ is the spreading factor.

The resulting baseband equivalent transmitted signal is given by:

$$
e(t)=\sum_{n} s_{n} \sum_{m=0}^{N_{s}-1} d_{m} g\left(t-m T_{c}-n T\right),
$$

where $T_{c}$ and $T$ are respectively the chip and symbol period and $g(t)$ is the impulse response of the root-raised cosine filter with rolloff factor equal to 0.22 in the case of the UMTS [1].

$h(t, \tau)$ denotes the overall impulse response of the multipath propagation channel with $L$ independent paths (WSSUS model):

$$
h(t, \tau)=\sum_{l=1}^{L} h_{l}(t) \delta\left(\tau-\tau_{l}(t)\right) .
$$

Each path is characterized by its time-varying delay $\tau_{l}(t)$ and channel coefficient $h_{l}(t)$.

The signal at the output of the matched filter is given by:

$$
r(t)=\sum_{l=1}^{L} h_{l}(t) \sum_{n} s_{n} \sum_{m=0}^{N_{s}-1} d_{m} R_{g}\left(t-m T_{c}-n T-\tau_{l}(t)\right)+\tilde{n}(t),
$$




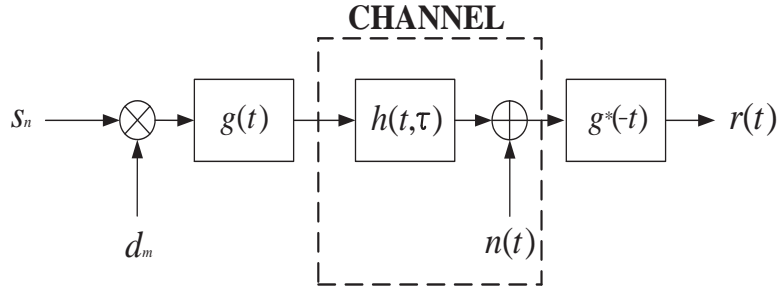

Fig. 1. Equivalent lowpass transmission system model.

where $\tilde{n}(t)$ represents the white gaussian noise $n(t)$ filtered by the matched filter and

$$
R_{g}(t)=\int_{-\infty}^{+\infty} g^{*}(\tau) g(t+\tau) d \tau
$$

is the total impulse response of the transmission and receiver filters.

Fig. 1 shows the equivalent lowpass transmission model considered in this paper.

\section{PARTICLE FILTERING FOR THE TIMING ERROR DETECTION}

In this paper, we propose to use the PF methods in order to track the delay of each individual path of the channel. We assume that the acquisition phase has allowed us to determine the number of the most significant paths and to roughly estimate their delay.

The PF methods are used to sequentially estimate timevarying quantities from measures provided by sensors. In general the physical phenomenon is represented by a state space model composed of two equations: the first equation describes the evolution of the unknown quantities called hidden state (evolution equation) and the second equation the relation between the measures called observations and the hidden state (observation equation). Given the initial distribution of the hidden state, the estimation of the hidden state at time $t$ based on the observations until time $t$ is known as Bayesian inference or Bayesian filtering. This estimation can be obtained through the knowledge of two distributions: the PPD of the sequence of hidden states from time 1 to time $t$ given the corresponding sequence of observations and the marginal distribution of the hidden state at time $t$ given the sequence of the observations until time $t$. Except in a few special cases including linear Gaussian state space models, it is impossible to express analytically these distributions. The PF methods provide a discrete approximation of the distributions, which can be updated when a new observation is available. The support of the distributions is discretized by particles, which are weighted samples evolving in time.
Tracking the delay of the individual channel paths can be interpreted as a Bayesian inference. The delays are the hidden state of the system and the model (3) of the received samples relating the observations to the delays represents the observation equation. We notice that this equation is nonlinear with respect to the delays and as a consequence, we cannot analytically estimate the delays. To overcome this nonlinearity, we propose to apply the PF methods. The PF methods have been previously considered for the delay estimation in DS-CDMA systems [2]: the PF methods are used to jointly estimate the data, the channel coefficients and the propagation delay. The delays of each channel path are supposed fixed and multiple of the sampling time. Then, these delays are known and only the propagation delay is estimated. In this paper, the approach is different. We suppose that each channel path has a time-varying delay, unknown at the receiver. This situation can represent for example an indoor wireless communication. We assume that the information symbols are known or have been estimated, since the channel is typically estimated using known pilot symbols contained in a specific channel, as the Common Pilot Channel (CPICH) of the UMTS. We apply the PF methods only to the estimation of the delays of each channel path, considering that the channel coefficients are known. In the presence of unknown channel coefficients, the PF-TED must be associated with a channel estimator.

The proposed PF-TED operates on samples from the matched filter output taken at an arbitrary sampling rate $1 / T_{s}$ (at least Nyquist sampling). Then, the samples are processed by means of interpolation and decimation in order to obtain intermediate samples at the chip rate $1 / T_{c}$. These samples are the input of the particle filter. The particle filter works at the symbol rate $1 / T$, exploiting all the information contained in the chips of a symbol period. The here proposed $\mathrm{PF}$ algorithm represents the adaptation of the PF methods to a DS-CDMA system.

We will consider that the delays evolve slowly in time and hence, that their evolution can be described with the following first order AutoRegressive (AR) model:

$$
\left\{\begin{array}{c}
\tau_{1}(n)=\alpha_{1} \tau_{1}(n-1)+v_{1}(n) \\
\vdots \\
\tau_{L}(n)=\alpha_{L} \tau_{L}(n-1)+v_{L}(n)
\end{array},\right.
$$

where $\alpha_{1}, \cdots, \alpha_{L}$ describe the possible time variation of the delays from a time to the next one and $v_{1}, \cdots, v_{L}$ are AWGN with zero mean and variance $\sigma_{v}^{2}$ [3].

The simulations give similar results for the Minimum Mean Square Error (MMSE) method and the Maximum A Posteriori (MAP) method. Hence, we choose to adopt the MMSE solution that is the simplest one in terms of computational complexity. The MMSE estimation is given by the 
following expectation:

$$
\hat{\tau}(n)=E[\tau(n) \mid r(1: n)],
$$

where $\tau(n)=\left\{\tau_{1}(n), \cdots, \tau_{L}(n)\right\}$ and $r(1: n)$ is the sequence of received samples from time 1 to $n$. The calculation of (6) involves the knowledge of the marginal distribution $p(\tau(n) \mid r(1: n))$. In order to obtain samples from the marginal distribution, we use the Sequential Importance Sampling (SIS) approach [7]. Applying the definition of the expectation, (6) can be expressed as follows:

$$
\hat{\tau}(n)=\int \tau(n) p(\tau(n) \mid r(1: n)) d \tau(n) .
$$

The aim of the SIS technique is to approximate the marginal distribution $p(\tau(n) \mid r(1: n))$ with weighted particles:

$$
p(\tau(n) \mid r(1: n)) \approx \sum_{i=1}^{N_{p}} \tilde{w}^{(i)}(n) \delta\left(\tau(n)-\tau^{(i)}(n)\right),
$$

where $N_{p}$ is the number of particles, $\tilde{w}^{(i)}(n)$ is the normalized importance weight at time $n$ associated with the particle $i$ and $\delta\left(\tau(n)-\tau^{(i)}(n)\right)$ denotes the Dirac delta centered in $\tau(n)=\tau^{(i)}(n)$.

Initially, the particles are in the same state $\tau^{(i)}(0)$ for $i=1, \ldots, N_{p}$ provided by the acquisition phase. The time evolution of the particles is achieved with an importance sampling distribution. For the transition from time $n-1$ to time $n$, the particles are drawn according to the importance function $\pi\left(\tau^{(i)}(n) \mid \tau^{(i)}(1: n-1), r(1: n)\right)$. Observing that the relation between the observations and the delays is nonlinear, we choose a prior importance function [7]:

$\pi\left(\tau^{(i)}(n) \mid \tau^{(i)}(1: n-1), r(1: n)\right)=p\left(\tau^{(i)}(n) \mid \tau^{(i)}(n-1)\right)$.

Considering that the noises $v_{l}(n)$ for $l=1, \cdots, L$ in (5) are Gaussian, the importance function for each delay $l$ is a Gaussian distribution with mean $\alpha_{l} \tau_{l}^{(i)}(n-1)$ and variance $\sigma_{v}^{2}$. To determine the positions of the particles at time $n$ from the positions at time $n-1$, each particle is drawn according to (9). The evaluation of the importance function for each particle at time $n$ enables the calculation of the importance weights [7]:

$w^{(i)}(n)=w^{(i)}(n-1) \frac{p\left(r(n) \mid \tau^{(i)}(n)\right) p\left(\tau^{(i)}(n) \mid \tau^{(i)}(n-1)\right)}{\pi\left(\tau^{(i)}(n) \mid \tau^{(i)}(1: n-1), r(1: n)\right)}$.

This expression represents the calculation of the importance weights if we only consider the samples of the received signal at the symbol rate. However, in a DS-CDMA system we have additional information provided by $N_{s}$ samples for each symbol period. Then we modify (10) taking into account the presence of a spreading sequence. Indeed, observing that the received samples are independent, the probability density $p\left(r(n) \mid \tau^{(i)}(n)\right)$ at the symbol rate can be written as:

$$
p\left(r(n) \mid \tau^{(i)}(n)\right)=\prod_{m=n N_{s}}^{(n+1) N_{s}-1} p\left(r(m) \mid \tau^{(i)}(n)\right) .
$$

Considering (3) at the chip rate and recalling the assumptions of known symbols, the probability density $p\left(r(m) \mid \tau^{(i)}(n)\right)$ is Gaussian with variance equal to the variance of the AWGN $\tilde{n}(t)$ in (3) and mean $\mu_{r}^{(i)}(m)$ obtained by:

$\mu_{r}^{(i)}(m)=\sum_{l=1}^{L} h_{l}(n) s_{n} \sum_{k=m-3}^{m+3} d_{k} R_{g}\left(m T_{c}-k T_{c}-n T-\tau_{l}^{(i)}(n)\right)$.

In order to reduce the computational complexity of the PFTED, in (12) we have assumed that the contribution of the raised cosine filter $R_{g}$ to the sum on the spreading sequence is limited to the previous 3 and next 3 samples.

The importance weights in (10) are normalized using the following expression:

$$
\tilde{w}^{(i)}(n)=\frac{w^{(i)}(n)}{\sum_{j=1}^{N_{p}} w^{(j)}(n)} .
$$

This algorithm presents a degeneracy phenomenon. After a few iterations of the algorithm, only one particle has a normalized weight almost equal to 1 and the other weights are very close to zero. This problem of the SIS method can be eliminated with a resampling of the particles. A measure of the degeneracy is the effective sample size $N_{e f f}$, estimated by:

$$
\hat{N}_{e f f}=\frac{1}{\sum_{i=1}^{N_{p}}\left(\tilde{w}^{(i)}(n)\right)^{2}} .
$$

When $\hat{N}_{\text {eff }}$ is below a fixed threshold $N_{\text {thres }}$, the particles are resampled according the weight distribution [7]. After each resampling task, the normalized weights are initialized to $1 / N_{p}$. Finally, by substitution of (8) into (7), we obtain at each time the MMSE estimate:

$$
\hat{\tau}(n)=\sum_{i=1}^{N_{p}} \tilde{w}^{(i)}(n) \tau^{(i)}(n) .
$$

\section{SIMULATION RESULTS}

In this section, we will compare the performance of the classical ELG-TED and the PF-TED. In order to demonstrate the gain achieved using the latter, we will consider an indoor scenario with a two Rayleigh path channel with the same average power on each path and a maximum Doppler frequency of $19 \mathrm{~Hz}$ corresponding to a mobile speed of 10 $\mathrm{Km} / \mathrm{h}$ for a carrier frequency of $2 \mathrm{GHz}$. The simulation setup is compatible with the UMTS standard. In these conditions, the time variations of the channel delays can be expressed 


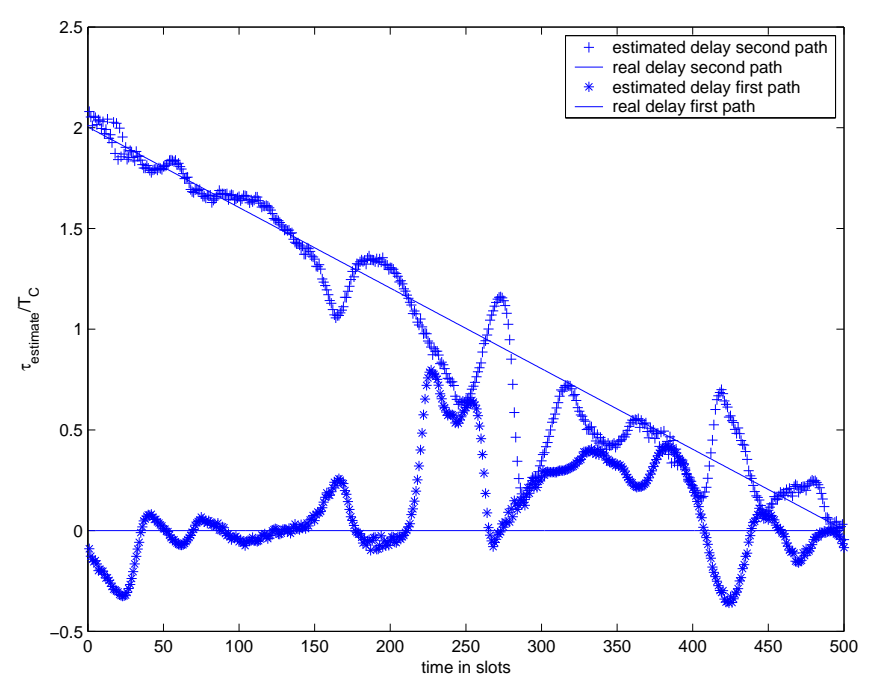

Fig. 2. Delay tracking with the classical ELG-TED.

by the model (5), with $\alpha_{1}=\cdots=\alpha_{L}=0.99999$ and $\sigma_{v}^{2}=10^{-5}$. We assume known symbols. A slot is composed of 40 pilots equal to 1 that are expanded into a chip level by a spreading factor of 64 . The spreading sequence is a PN sequence changing at each symbol. We have choose a two-path channel with delay of the first path fixed to 0 and delay of the second path decreasing linearly from $2 T_{c}$ to 0 over a simulation time of $0.333 \mathrm{~s}$ corresponding to 500 slots.

Fig. 2 gives a representative example of the evolution of the two estimated delays using two ELG-TED. As soon as the difference between the two delays is lower than $1 T c$, the detectors continues to distinguish the two paths, but it is unable to track the two delays. On the other hand, as shown in Fig. 3 the proposed detector can track almost perfectly the two paths. These results have been obtained using a particle detector with 10 particles and assuming that $E_{s} / N_{o}=10 d B$, where $E_{s}$ is the energy per symbol and $N_{o}$ is the unilateral spectral power density.

\section{CONCLUSIONS}

In this paper we have proposed to use the PF methods in order to track the delay of the different paths of a channel. We have assumed that an acquisition phase has already provided an initial estimation of these delays. We have applied the PF methods to solve the problem of the classical ELGTED when the paths are very close. We have shown that the PF-TED can continue to track paths even when the ELGTED loses them. Future research fields will be the analysis of the computational complexity of the PF-TED in the presence of unknown channel coefficients and the extension of the PF methods to the acquisition phase in the case of unresolvable paths.

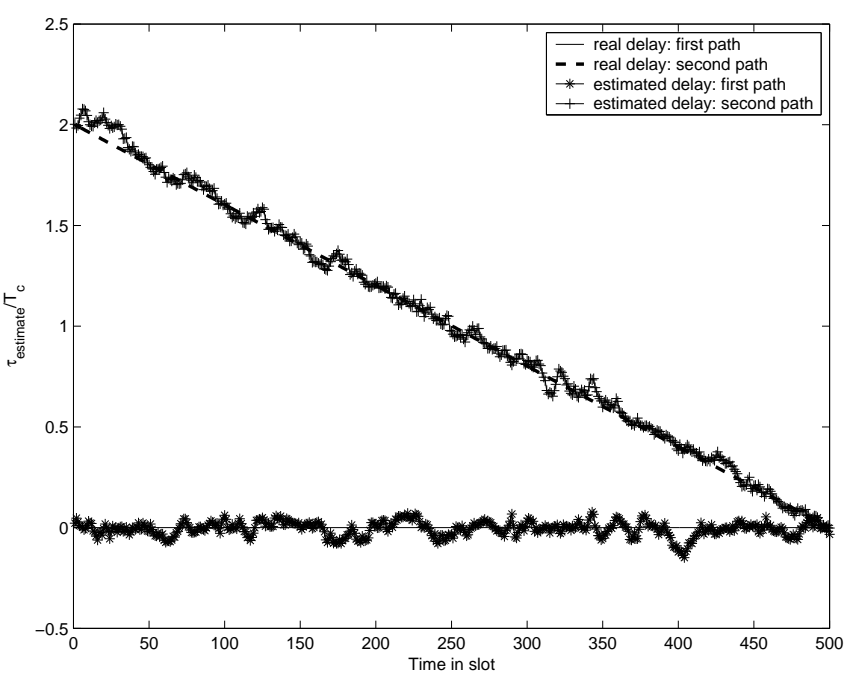

Fig. 3. Delay tracking with the PF-TED.

\section{REFERENCES}

[1] 3GPP technical specification group radio access network, spreading and modulation (FDD), 3G TS 25.213 version 3.3.0, decembre 2000.

[2] E. Punskaya, A. Doucet and W. J. Fitzgerald, "On the use and misuse of particle filtering in digital communications," EUSIPCO 2002, Toulouse, Sept. 2002.

[3] R. A. Ilits, "Joint estimation of PN code delay and multipath using the extended Kalman filter," IEEE Trans. on Com., Vol. 38, pp. 1677-1685, Oct 1990.

[4] V. Aue, G. P. Fettweis, "A non-coherent traching scheme for the Rake receivier that can cope with unresolvable multipath," Proc. IEEE International Conference on Communications, June. 1999.

[5] R. De Gaudenzi, "Direct-sequence spread spectrum chip tracking in the presence of unresolvable multipath components," IEEE Trans. on Vehical Tech., Vol. 48, pp. 1573-1583, Sept 1999.

[6] A. Doucet, J. F. G. de Freitas and N. J. Gordon, Sequential Monte Carlo methods in practice. New York: Springer-Verlag, 2001.

[7] A. Doucet, S. Godsill and C. Andrieu, "On sequential Monte Carlo sampling methods for Bayesian filtering," Statistics and Computing, Vol. 10, No. 3, pp. 197-208, 2000.

[8] N. J. Gordon, D. J. Salmond and A. F. M. Smith, "Novel approach to nonlinear/non-Gaussian Bayesian state estimation," IEE Proc., Vol. 140(2), pp. 107-113, 1993. 